\title{
Reliability Modeling and Analysis for Typical System with Multi- components via Possibility Theory
}

\author{
Jiaojiao Ren ${ }^{1, ~ a ~}$, Yuping Zhang ${ }^{1, b}$, Yuhua Wei ${ }^{1, c}$, Chenfang Zhang ${ }^{1, d}$, \\ Biao Qin ${ }^{2, e}$ \\ ${ }^{1}$ School of Automation Engineering, University of Electronic Science and Technology of China, \\ Chengdu, \\ ${ }^{2}$ School of Mathematics Science, University of Electronic Science and Technology of China, \\ Chengdu \\ ajiaojiaoren06@163.com, byp_002@163.com, cwei_wj_wyh@163.com, dhmyzzcf@126.com, ${ }^{\mathrm{e}}$ qinbi \\ aolixin@163.com
}

Keywords: Typical systems; Possibilistic reliability; Possibility measures; Reliability model.

\begin{abstract}
In this note, system Posbist reliability theories are improved due to the distribution functions of the lifetime of system components are different. The simulations are given to compare Posbist reliability between different typical systems.
\end{abstract}

\section{Introduction}

With the increasing accuracy and automation of mechanical equipment, reliability has been a very important concept in engineering as well as a primary quality index of guaranteeing stable performances. A remarkably successful tool is the traditional probabilistic reliability method [1] which can be used to deal with uncertainty. In fact, the lack of sample date is a very common occurrence because the complex of mechanical equipment's structure and the stern working condition. And there exist vast subjective information and epistemic uncertainty in initial data. The traditional stochastic method has shown inadaptability in solving this problem [2].

The integration of possibility theory and reliability model have greater processing advantages for large complex system, equipment and parts, which is difficult to acquire the necessary statistics. For some parameter without probability statistic character, Subjective evaluation method have higher credibility compared to probability statistics method. Especially, Posbist reliability theory. Posbist reliability models have been constructed in [3]. In [4], the authors remove the requirement of irreparable, Posbist reliability models of repairable system were established. The authors in [5] analyzed system Posbist reliability by considering the system equation as functional equation. Based on possibility assumption and binary-state assumption, the Posbist reliability models were given in [6,7], which the distribution functions of the lifetime of system components were assumed as the same. To best of our knowledge, there are no results about Posbist reliability models under different distribution function.

Motivated by the above mentioned discussion, we discuss the problem of reliability modeling and analysis for typical system with multi-components using Posbist reliability theory, which is based on possibility assumption and binary-state assumption. In this paper, System Posbist reliability models are established, which the distribution functions are different. The examples are given to compare Posbist reliability between different typical systems.

\section{Preliminaries}

\section{Definition 1. (System lifetime) [6]}

Let fuzzy variable $X$ be a real valued function defined on a possibility space $\left(U, \Phi, P_{o s s}\right)$, i.e. $X: U \rightarrow R=(-\infty,+\infty)$, with the membership function $\mu_{X}: U \rightarrow[0,1]$, then the lifetime of a system is 
a nonnegative real-valued fuzzy variable, i.e. $X: U \rightarrow R^{+}=(0,+\infty)$, with possibility distribution function

$$
\pi_{X}(x)=\mu_{X}(x)=P_{\text {oss }}(X=x), x \in R^{+}
$$

\section{Definition 2. (Posbist reliability) [7]}

The Posibist reliability of a system is defined as the possibility that the system performs its assigned functions during a predefined exposure period under a given conditions, which is denoted as

$$
R_{e}(t)=P_{\text {oss }}(X>t)=\sup _{u>t} P_{\text {oss }}(X=u)=\sup _{u>t} \mu_{X}(u), t \in R^{+}
$$

\section{Main results}

Given a possibility space $\left(U, \Phi, P_{\text {oss }}\right)$,the fuzzy variables $X_{1}, X_{2}, \cdots, X_{n}$ are said to be mutually unrelated, we assume the lifetime of the system $X_{i}(i=1,2, \cdots, n)$ as a symmetrical Gaussian fuzzy variable with the following distribution function $\left(m_{i}, h_{i}>0\right.$ and $\left.i=1,2, \ldots, n\right)$

$$
\mu_{X_{i}}(x)=\left\{\begin{array}{ll}
\exp \left(-\left(\frac{m_{i}-x}{h_{i}}\right)^{2}\right), & x \leq m_{i} \\
\exp \left(-\left(\frac{x-m_{i}}{h_{i}}\right)^{2}\right), & x \leq m_{i}
\end{array} .\right.
$$

\subsection{The Posbist reliablity models of series system}

We consider a series system consisting of $n$ components, the lifetime of component is $X_{i}(i=1,2, \cdots, n)$ and the lifetime of system is $X=\min \left\{X_{1}, X_{2}, \cdots, X_{n}\right\}$, we have a assumption that $h_{i}=h$. Without loss of generality, we assume $m_{1} \leq m_{2} \leq \ldots \leq m_{n}$.

Theorem 1. Given a series system consisting of $n$ components, the lifetime of system and component is $X_{i}(i=1,2, \cdots, n)$ and $X=\min \left\{X_{1}, X_{2}, \cdots, X_{n}\right\}$, which are defined in possibility space $\left(U, \Phi, P_{\text {oss }}\right)$. Assume that $X_{1}, X_{2}, \ldots, X_{n}$ is mutually unrelated and its continuous possibility distribution function $\mu_{X_{i}}(x)$ is a fuzzy variable with the normality and strictly convex feature. Then exist a positive number $m_{1}$, the Posbist reliability of the series system consisting of $n$ components can be acquired as

$R_{s}(x)=\left\{\begin{array}{ll}1, & x \leq m_{1} \\ \exp \left(-\left(\frac{x-m_{1}}{h}\right)^{2}\right), & x>m_{1}\end{array}\right.$.

\subsection{The Posbist reliablity models of parallel system}

We consider a parallel system consisting of $n$ components, the lifetime of component is $X_{i}(i=1,2, \cdots, n)$ and the lifetime of system is $X=\max \left\{X_{1}, X_{2}, \cdots, X_{n}\right\}$, under the same assumption.

Theorem 2. Given a parallel system consisting of $n$ components, the lifetime of system and component is $X_{i}(i=1,2, \cdots, n)$ and $X=\max \left\{X_{1}, X_{2}, \cdots, X_{n}\right\}$, which are defined in possibility space $\left(U, \Phi, P_{\text {oss }}\right)$. Assume that $X_{1}, X_{2}, \ldots, X_{n}$ is mutually unrelated and its continuous possibility distribution function $\mu_{X_{i}}(x)$ is a fuzzy variable with the normality and strictly convex feature. Then exist a positive number $m_{n}$, the Posbist reliability of the parallel system consisting of $n$ components can be acquired as

$$
R_{p}(x)=\left\{\begin{array}{ll}
1, & x \leq m_{n} \\
\exp \left(-\left(\frac{x-m_{n}}{h}\right)^{2}\right), & x>m_{n}
\end{array} .\right.
$$




\subsection{The Posbist reliablity models of series-parallel system}

We consider a series-parallel system consisting of $m$ series subsystem, and each subsystem is parallel system consisting of $n$ components, which is shown in Fig. 1, the lifetime of component is $X_{i}(1 \leq i \leq m n)$ and under the same assumption.

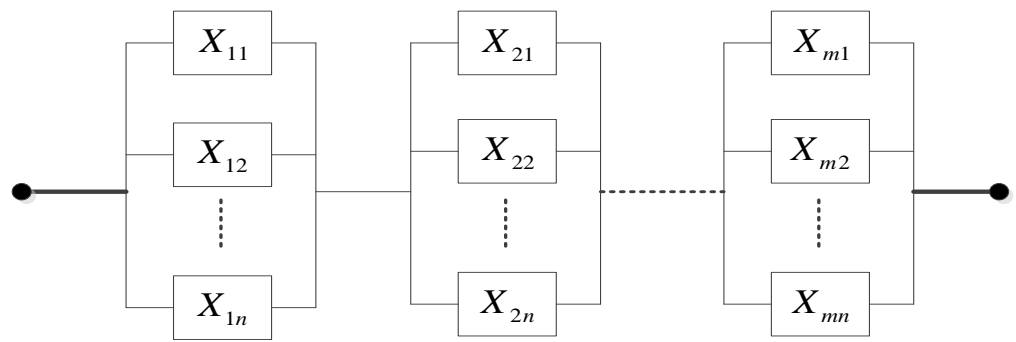

Figure 1. The logic block diagram of a series-parallel system.

Theorem 3. Given a series-parallel system consisting of $m n$ components, the lifetime of system and component is $X_{1}, X_{2}, \ldots, X_{m n}$ and $X$, which are defined in possibility space $\left(U, \Phi, P_{\text {oss }}\right)$. Assume that $X_{1}, X_{2}, \ldots, X_{m n}$ is mutually unrelated and its continuous possibility distribution function $\mu_{X_{i}}(x)$ is a fuzzy variable with the normality and strictly convex feature. Then exist a positive number $L$, such that the Posbist reliablity models of series-parallel system can be expressed as

$R_{s p}(x)=\left\{\begin{array}{ll}1, & x \leq L \\ \exp \left\{-\left(\frac{x-L}{h}\right)^{2}\right\} & , x>L\end{array}\right.$.

Where $L=\min \left\{K_{1}, K_{2}, \cdots, K_{m}\right\}, K_{i}=\max \left\{m_{i 1}, m_{i 2}, \cdots, m_{i n}\right\}$.

\subsection{The Posbist reliablity models of parallel -series system}

We consider a parallel- series system consisting of $m$ parallel subsystem, and each subsystem is series system consisting of $n$ components, which is shown in Fig. 2, under the same assumption.

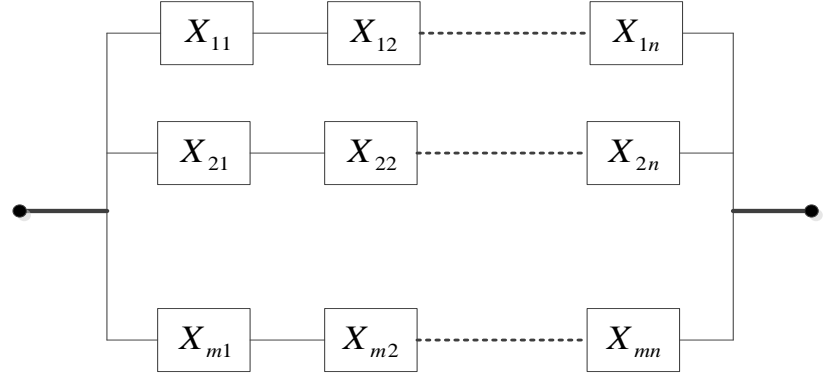

Figure 2. The logic block diagram of a parallel-series system.

Theorem 4. Given a parallel-series system consisting of $m n$ components, the lifetime of component and system is $X_{1}, X_{2}, \ldots, X_{m n}$ and $X$, which are defined in possibility space $\left(U, \Phi, P_{\text {oss }}\right)$. Assume that $X_{1}, X_{2}, \ldots, X_{m n}$ is mutually unrelated and its continuous possibility distribution function $\mu_{X_{i}}(x)$ is a fuzzy variable with the normality and strictly convex feature. Then exist a positive number $Q$, such that the Posbist reliablity models of parallel-series system can be expressed as

$R_{p s}(x)=\left\{\begin{array}{ll}1, & x \leq Q \\ \exp \left\{-\left(\frac{x-Q}{h}\right)^{2}\right\} & , x>Q\end{array}\right.$.

Where $Q=\max \left\{P_{1}, P_{2}, \cdots, P_{m}\right\}, P_{i}=\min \left\{m_{i 1}, m_{i 2}, \cdots, m_{i n}\right\}$. 


\section{Numerical example}

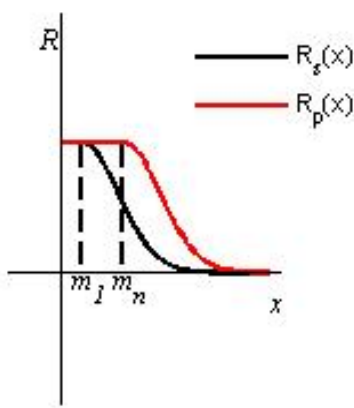

Figure.3

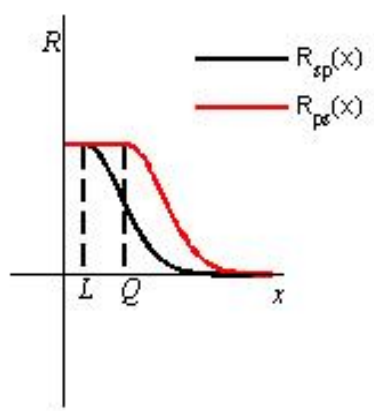

Figure. 4

4.1 The comparison of Posbist reliability of a series system and parallel system

Assume that a series system and parallel system consist of $n$ components, and the lifetime distribution function of components are the same one. According to Theorem 1 and Theorem 2, the Posbist reliability of a series system and parallel system are shown in Fig. 3.

4.2 The comparison of Posbist reliability of a series-parallel system and parallel-series system

Assume that a series-parallel system and parallel-series system consist of $m n$ components, and the lifetime distribution function of components are the same one. According to Theorem 3 and Theorem 4, the Posbist reliability of a series-parallel system and parallel-series system are shown in Fig. 4.

\section{Conclusions}

In this paper, we remove the requirement of the lifetime distribution function must be the same. The Posbist reality models of a series system, parallel system, series-parallel system and parallelseries system are established, which the distribution functions of components are different. Finally, numerical examples are given to compare the Posbist reality between different system.

\section{Acknowledgements}

The research work was supported by the National Defense Pre-Research Foundation of China under Grant No. 9140A27040213DZ02403 and the Fundamental Research Funds for the Central Universities (No. ZYGX2014J070).

\section{References}

[1]. Mu Zhi-Zhong, Zhu Wen-Yu. Mechanical reliability design. Beijing: China Machine Press, 1993.

[2]. Zhao Guo-Fan. Engineering structure reliability theory and application. Dalian: Dalian university of technology press, 1996.

[3]. Cai K Y, Wen C Y, Zhang M L. Fuzzy variables as a basis for a theory of fuzzy reliability in the possibility context. Fuzzy Sets and Systems, 42 (2), pp. 145-172, 1991.

[4]. Utkin L V. Fuzzy reliability of repairable systems in the possibility context. Mieroeleetronies and Reliability, 34 (12), pp. 1865-1876, 1994.

[5]. Utkin LV, Gurov S V. A general formal approach for fuzzy reliability analysis in the possibility context, Fuzzy Sets and Systems, 83 (2), pp. 203-213, 1996.

[6]. Cai K Y. Introduction to Fuzzy Reliability. Boston: Kluwer academic Publishers, 1996.

[7]. He L P. Mechanical system reliability analysis and evaluation based on the probability measure: PhD Dissertation, Liaoning: Dalian university of technology, 2010. 